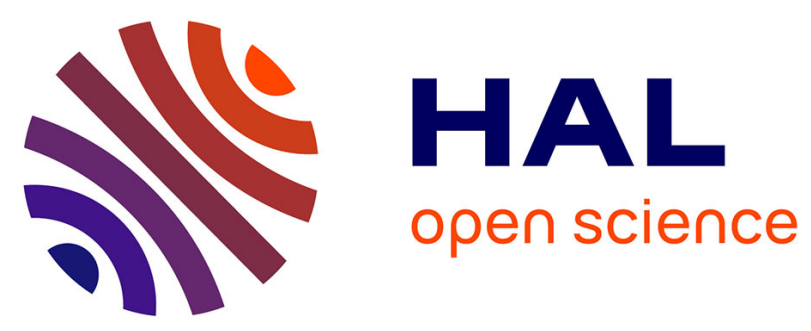

\title{
FER/Impact : Logiciel de simulation numérique des problèmes d'impact
}

\author{
Zhi-Qiang Feng, Benoit Magnain, Jean-Michel Cros
}

\section{To cite this version:}

Zhi-Qiang Feng, Benoit Magnain, Jean-Michel Cros. FER/Impact: Logiciel de simulation numérique des problèmes d'impact. Revue Européenne de Mécanique Numérique/European Journal of Computational Mechanics, 2006, 15 (1-3), pp.175-186. 10.3166/remn.15.175-186 . hal-01179165

\section{HAL Id: hal-01179165 \\ https://hal.science/hal-01179165}

Submitted on 3 Jul 2018

HAL is a multi-disciplinary open access archive for the deposit and dissemination of scientific research documents, whether they are published or not. The documents may come from teaching and research institutions in France or abroad, or from public or private research centers.
L'archive ouverte pluridisciplinaire HAL, est destinée au dépôt et à la diffusion de documents scientifiques de niveau recherche, publiés ou non, émanant des établissements d'enseignement et de recherche français ou étrangers, des laboratoires publics ou privés. 


\section{FER/Impact : logiciel de simulation numérique des problèmes d'impact}

\section{Zhi-Qiang Feng — Benoît Magnain — Jean-Michel Cros}

Laboratoire de Mécanique et d'Énergétique d'Évry

Université d'Évry - Val d'Essonne

40, rue du Pelvoux

F-91020 Évry Cedex

\{feng,magnain,cros\}@iup.univ-evry.fr

RÉSUMÉ. Cet article présente le code de calcul FER/Impact pour simuler des problèmes de contact/impact, entre solides déformables ou non, avec ou sans frottement. Ce code de calcul combine la méthode du bi-potentiel et un schéma d'intégration en temps du premier ordre. Les différentes possibilités de simulation sont indiquées et l'efficacité est illustrée par quelques exemples.

ABSTRACT. This paper presents the program FER/Impact for the modelling of contact/impact problems between deformable bodies with or without friction. This program combines the bi-potential method and a time-stepping scheme of first order. Different possibilities and the efficiency of the code are illustrated from several numerical examples.

MOTS-CLÉS : impact, contact, frottement, éléments finis.

KEYWORDS: impact, contact, friction, finite element. 


\section{Introduction}

La famille des logiciels FER (Finite Element Research), développée par le groupe MDS (Modélisation en Dynamique des Structures) du Laboratoire de Mécanique et d'Énergétique d'Évry (LMEE) permet de traiter de nombreux problèmes en mécanique des solides et des structures par la méthode des éléments finis (Feng 2000). Récemment, un nouvel outil nommé FER/Impact a été ajouté afin de résoudre les problèmes d'impact. La résolution de ces problèmes fait apparaître deux grandes difficultés qui sont, d'une part, le problème du contact avec frottement et, d'autre part, l'intégration temporelle des équations du mouvement. Au cours des deux dernières décennies, des avancées importantes ont été accomplies dans l'analyse des problèmes de contact par la méthode des éléments finis. La méthode de pénalisation ou de régularisation est la plus couramment utilisée dans les logiciels industriels (ANSYS, RADIOSS,...) pour traiter les problèmes de contact. Mais pour ce type de méthode, les conditions de contact et les lois de frottement ne sont pas satisfaites exactement. De plus, il est délicat pour les utilisateurs de choisir le facteur de pénalité adéquat. Les expériences ont montré que ces méthodes présentent des inconvénients en ce qui concerne la stabilité et la précision numérique, en particulier pour tout ce qui touche à la simulation des phénomènes de frottement. Pour pallier ces insuffisances, une méthode du bi-potentiel a été développée (De Saxcé et al. 1998) et appliquée avec succès aux problèmes du contact en statique. Le logiciel FER/Impact met en œuvre une extension de cette méthode à l'analyse des problèmes d'impact, on en trouvera une description détaillé dans (Feng et al. 2005a). Pour la résolution des problèmes d'impact, les schémas d'intégration du second ordre sont les plus utilisés (Armero et al. 1998). Dans les schémas du second ordre, l'accélération est supposée constante ou alors elle varie de façon linéaire. Or, pour les problèmes d'impact, la vitesse et l'accélération ne sont pas continues en raison du changement brutal des conditions de contact (impact, rebond,...). Dans ces conditions, les algorithmes du second ordre peuvent conduire à de graves erreurs (Armero et al. 1998). Il est possible de corriger ces problèmes (Laursen 2002), via par exemple des techniques de régularisation mais qui conduisent à des développements complexes. Une alternative consiste à employer des algorithmes du premier ordre (Jean 1989, Jean 1999). Ainsi, l'algorithme implanté dans le code de calcul FER/Impact combine la méthode du bi-potentiel et un schéma d'intégration temporelle du premier ordre. Dans (Feng et al. 2005c) on trouvera une comparaison de la méthode proposée avec les algorithmes disponibles dans le code de calcul ANSYS.

L'article s'articule comme suit; une première partie décrit succinctement les méthodes de discrétisation et d'approximation employées. Une seconde partie résume les principales possibilités de modélisation suivie d'une partie qui porte sur les techniques informatiques utilisées. Enfin, plusieurs exemples mettent en évidence le potentiel du code de calcul sur différents types de problèmes (2D, 3D) et types d'impact (déformable/rigide, déformable/déformable) avec plusieurs lois de comportement (élastique, hyper-élastique) et prise en compte ou non du frottement. 


\section{Description des algorithmes}

La résolution du problème de dynamique, qui nous intéresse, consiste à prendre en compte, par rapport au cas sans contact, les réactions au niveau des surfaces de contact. Ainsi, après discrétisation spatiale par la méthode des éléments finis (approche Lagrangienne totale), un problème de contact en dynamique et dans le cas non linéaire s'écrit sous la forme de l'équation d'équilibre suivante :

$$
\mathbf{M} \ddot{\mathbf{u}}=\mathbf{F}+\mathbf{R}_{c}, \quad \text { où } \mathbf{F}=\mathbf{F}_{\text {ext }}+\mathbf{F}_{\text {int }}-\mathbf{C} \dot{\mathbf{u}}
$$

Les vecteurs $\mathbf{F}_{\text {ext }}, \mathbf{F}_{\text {int }}$ et $\mathbf{R}_{c}$ représentent respectivement les forces externes, internes, et de contact. $\mathbf{M}$ est la matrice de masse du système et $\mathbf{C}$ la matrice d'amortissement. Pour réaliser l'intégration de l'équation [1], entre deux instants consécutifs $t$ et $t+\Delta t$, il est classique d'utiliser l'algorithme du second ordre de Newmark. Cependant, dans les problèmes d'impact, les approximations d'ordre supérieur ne signifient pas nécessairement une meilleure précision. En effet, lors du changement brutal des statuts de contact, la vitesse et l'accélération ne sont pas continues, et la régularité excessive peut conduire à des erreurs importantes (Armero et al. 1998). Pour cette raison, Jean (Jean 1989, Jean 1999) a proposé un algorithme du premier ordre qui est employé dans ce travail. Cet algorithme est basé sur les approximations suivantes :

$$
\begin{aligned}
& \mathbf{u}^{t+\Delta t}-\mathbf{u}^{t}=\Delta t\left((1-\theta) \dot{\mathbf{u}}^{t}+\theta \dot{\mathbf{u}}^{t+\Delta t}\right) \\
& \int_{t}^{t+\Delta t} \mathbf{F} d t=\Delta t\left((1-\xi) \mathbf{F}^{t}+\xi \mathbf{F}^{t+\Delta t}\right)
\end{aligned}
$$

où $0 \leq \xi \leq 1 ; \quad 0 \leq \theta \leq 1$. Pour le processus itératif, les valeurs au temps $t+\Delta t$ sont remplacées par les valeurs à l'itération $i+1$; soit par exemple, $\mathbf{F}^{t+\Delta t}=\mathbf{F}^{i+1}$. L'approximation standard de $\mathbf{F}^{i+1}$ donne :

$$
\mathbf{F}^{i+1}=\mathbf{F}_{i n t}^{i}+\frac{\partial \mathbf{F}}{\partial \mathbf{u}}\left(\mathbf{u}^{i+1}-\mathbf{u}^{i}\right)+\frac{\partial \mathbf{F}}{\partial \dot{\mathbf{u}}}\left(\dot{\mathbf{u}}^{i+1}-\dot{\mathbf{u}}^{i}\right)=\mathbf{F}_{i n t}^{i}-\mathbf{K}^{i} \Delta \mathbf{u}-\mathbf{C}^{i} \Delta \dot{\mathbf{u}}
$$

où $\mathbf{K}^{i}$ représente la matrice de rigidité tangente à l'itération $i$. Finalement, nous obtenons la forme récursive en déplacement suivante :

$$
\begin{gathered}
\left\{\begin{array}{c}
\overline{\mathbf{K}}^{i} \Delta \mathbf{u}=\overline{\mathbf{F}}^{i}+\overline{\mathbf{F}}_{a c c}^{i}+\mathbf{R}_{c}^{i+1} \\
\mathbf{u}^{i+1}=\mathbf{u}^{i}+\Delta \mathbf{u}
\end{array}\right. \\
\operatorname{avec}\left\{\begin{array}{c}
\overline{\mathbf{K}}^{i}=\xi \mathbf{K}^{i}+\frac{\xi}{\theta \Delta t} \mathbf{C}^{i}+\frac{1}{\theta \Delta t^{2}} \mathbf{M} \\
\overline{\mathbf{F}}_{a c c}^{i}=-\frac{1}{\theta \Delta t^{2}} \mathbf{M}\left\{\mathbf{u}^{i}-\mathbf{u}^{t}-\Delta t \dot{\mathbf{u}}^{t}\right\} \\
\overline{\mathbf{F}}^{i}=(1-\xi)\left(\mathbf{F}_{\text {int }}^{t}+\mathbf{F}_{\text {ext }}^{t}\right)+\xi\left(\mathbf{F}_{\text {int }}^{i}+\mathbf{F}_{\text {ext }}^{t+\Delta t}\right)
\end{array}\right.
\end{gathered}
$$

Finalement, à la fin de chaque pas de temps, la vitesse est réactualisée par :

$$
\dot{\mathbf{u}}^{t+\Delta t}=\left(1-\frac{1}{\theta}\right) \dot{\mathbf{u}}^{t}+\frac{1}{\theta \Delta t}\left(\mathbf{u}^{t+\Delta t}-\mathbf{u}^{t}\right)
$$


On cherche, pour le cas d'impact sans frottement, à vérifier le principe de conservation de l'énergie et à quantifier l'énergie dissipée lors des impacts avec frottement. Pour cela, il est possible d'agir sur les paramètres du schéma d'intégration. Pour $\theta=0,5$ ce schéma correspond à la règle du point milieu implicite. Simo et Wong (Simo et al. 1991) ont montré que ce schéma préserve l'énergie totale et l'équilibre pour les problèmes dynamiques sans contact. Dans la suite, on prendra $\theta=\xi=0,5$. Numériquement, pour ces valeurs, le schéma d'intégration est stable et préserve l'énergie totale dans le cas sans frottement. Les exemples numériques vont montrer que ce choix permet également d'assurer la stabilité pour les problèmes d'impact avec frottement. Pour des valeurs de $\xi$ et de $\theta$ plus petites ou plus grandes, on constate l'apparition d'une dissipation numérique (Feng et al. 2005a).

La dernière difficulté consiste à calculer la force de réaction de contact $\mathbf{R}_{c}^{i+1}$ qui apparaît dans [5]. Cette force est obtenue par une méthode du bi-potentiel proposée par De Saxcé et Feng (De Saxcé et al. 1991, Feng 1995, De Saxcé et al. 1998). Avec cette méthode du bi-potentiel le problème du contact avec frottement est traité dans un système réduit par un algorithme d'Uzawa avec une seule phase de prédictioncorrection sur le cône de frottement. Cette dernière méthode n'utilise qu'un seul principe variationnel sur le déplacement et une seule inégalité sur le contact unilatéral et le frottement. Cette approche a été appliquée avec succès aux problèmes de contact avec frottement pour des cas quasi statiques (Feng 1995, Feng 1998, Feng et al. 2003).

\section{Domaines d'application de FER/Impact}

Les différentes caractéristiques du code et les possibilités de simulation sont les suivantes :

- méthode des éléments finis : formulation lagrangienne totale;

- loi de comportement : élastique linéaire ou hyper-élastique (Blatz-Ko...);

- problèmes en grandes transformations (grands déplacements, grandes rotations et grandes déformations);

- problèmes 2D ou 3D : différents éléments finis ont été implantés afin de pouvoir résoudre des problèmes $2 \mathrm{D}$ (élements à 3 et 4 nœuds) ou $3 \mathrm{D}$ (éléments tétraèdre à 4 nœuds et brique à 8 nœuds);

- contact/impact entre plusieurs solides déformables ou plusieurs solides déformables et rigides : le code incorpore une méthode automatique de détection du contact entre les solides qui nécessite simplement la définition, préalable au calcul, des zones susceptibles d'être en contact;

- prise en compte du frottement : loi de frottement de type Coulomb (isotropre et orthotrope). Il convient d'ajouter que le contact unilatéral et le frottement sont couplés par l'intermédiaire du bi-potentiel. Un point essentiel de la méthode du bi-potentiel est que le correcteur peut être analytiquement trouvé en ce qui concerne les trois statuts possibles du contact (contact avec adhérence, pas de contact, contact avec glissement). Il est important de souligner que la formule explicite de correction est valable à la 
fois pour les problèmes de contact $2 \mathrm{D}$ et $3 \mathrm{D}$ avec frottement de Coulomb et permet d'obtenir des résultats très stables et précis ;

- schéma d'intégration en temps : le schéma du premier ordre (méthode du point milieu) est privilégié, mais afin de mener des études comparatives, un schéma du second ordre de type Newmark est également implanté.

\section{Organisation générale du code}

Le code prototype FER/Impact (environ 4000 lignes), est principalement écrit en $\mathrm{C}++$ suivant un modèle de programmation orienté objet. Les sous-programmes de calcul (factorisation, résolution de système linéaire,...) sont écrits en Fortran et exploitent les librairies mathématiques optimisées (BLAS, LAPACK...) dans un souci de performance. Le code est de plus portable puisque des simulations ont été conduites sur différents types de machines mono ou multi-processeur (station Linux, IBM SP4, SGI $3800, \ldots$.$) . Le code est organisé de façon très modulaire, ce qui permet d'ajouter de nou-$ velles fonctions de manière très simple, comme par exemple l'ajout d'un schéma du second ordre ou, plus délicat, la prise en compte du frottement orthotrope ou d'une loi de comportement hyper-élastique. Par ailleurs, lorsque 1'on traite des problèmes faisant intervenir plusieurs solides élastiques en interaction (chocs de plusieurs billes par exemple), le parallélisme est évident, chaque bille pouvant être traitée indépendamment, l'interaction se situant uniquement sur les zones de contact. Cela s'apparente à des méthodes de décomposition de domaine. Pour le moment, l'extension des possibilités du code (nouvelles lois de comportement, nouveaux éléments) est privilégiée. Aussi, le parallélisme se limite à la construction du problème condensé sur les zones de contact.

\section{Résultats numériques}

\subsection{Impact en grandes déformations : analyse détaillée}

Cet exemple (figure 1) consiste en l'étude de l'impact d'un cylindre en mousse polyuréthanne sur deux plans rigides obliques symétriques qui constituent une rainure en forme de "v". Les caractéristiques de ce problèmes sont les suivantes : loi de comportement de type Blatz-Ko (Feng et al. 2003), module de cisaillement $G=3 \mathrm{MPa}$, masse volumique $\rho=700 \mathrm{~kg} / \mathrm{m}^{3}$, vitesse initiale $v_{y}=-30 \mathrm{~m} / \mathrm{s}$. Le rayon du cylindre $R$ est égal à $0,01 \mathrm{~m}$. La simulation se déroule sur $310^{-3} \mathrm{~s}$ et les paramètres de l'algorithme sont $\Delta t=10^{-5} \mathrm{~s}, \xi=\theta=0,5$. Le problème est traité en déformations planes et le maillage du cylindre (figure 1) comprend 209 nœuds et 192 éléments finis à quatre nœuds. La position initiale du cylindre est repérée par son centre, noté $O(0,0$; $0,03)$. Le bloc rigide à droite est défini par quatre points : $A(0,005 ; 0,0), B(0,015$; $0,0), C(0,015 ; 0,035)$ et $D(0,012 ; 0,035)$. 


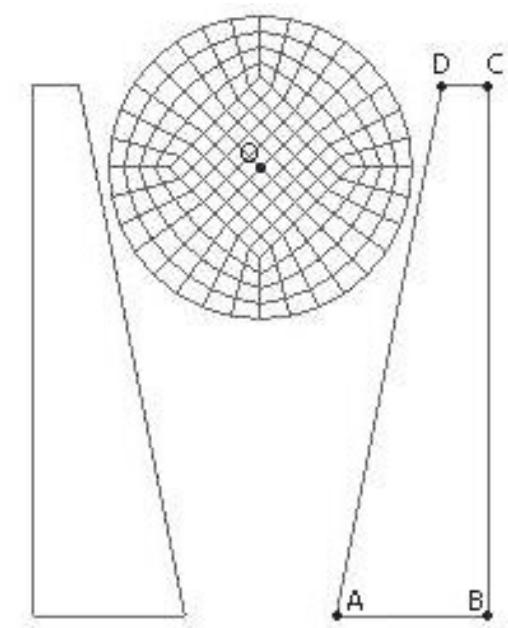

Figure 1. Impact entre un solide hyper-élastique et des surfaces rigides

Trois simulations (cas A, B et C) qui correspondent à différents coefficients de frottement (tableau 1) sont effectuées. La figure 2 montre la distribution des contraintes équivalentes de von Mises dans le cylindre au moment où celui-ci atteint sa position la plus basse et lorsque l'énergie cinétique $E_{c}$ est égale à zéro (figures 5 à 7). Le temps de simulation pour obtenir la valeur maximale de la contrainte équivalente de von Mises, dans les trois cas, est reporté dans le tableau 1. On constate que lorsque le coefficient de frottement augmente, la valeur de la contrainte diminue ainsi que la déformation du solide (figure 2).
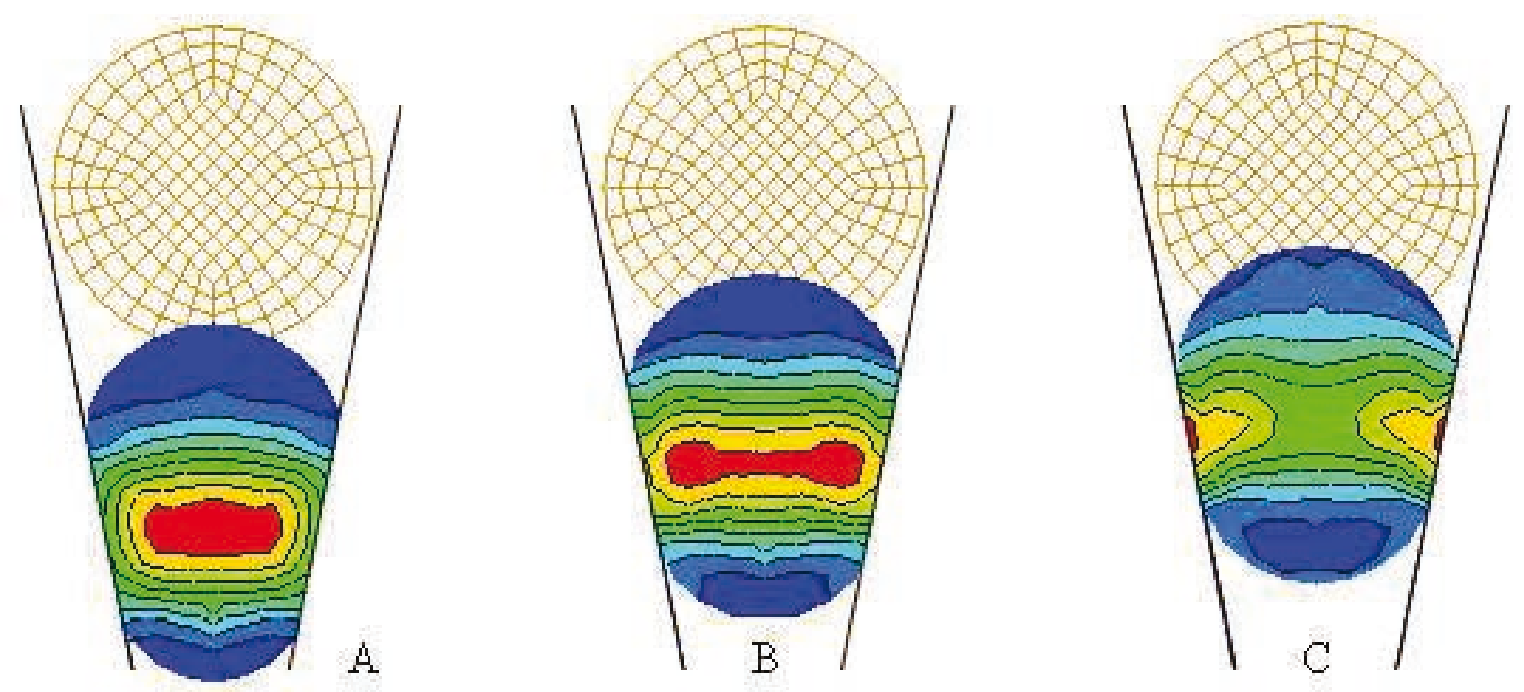

Figure 2. Isovaleurs des contraintes équivalentes de von Mises

\begin{tabular}{|c|c|c|}
\hline Cas & à l'instant $(\mathrm{ms})$ & $\sigma_{\max }(\mathrm{MPa})$ \\
\hline $\mathrm{A}: \mu=0,0$ & 0,87 & 8,192 \\
$\mathrm{~B}: \mu=0,2$ & 0,70 & 4,523 \\
$\mathrm{C}: \mu=0,4$ & 0,61 & 4,396 \\
\hline
\end{tabular}

Tableau 1. Influence du coefficient de frottement 
La figure 2 montre également, pour les trois cas, des différences sur la localisation des contraintes maximales. Dans le cas A, le cylindre descend très bas et se déforme beaucoup. Par conséquent la contrainte est la plus élevée. Lorsque le frottement augmente, le cylindre descend de moins en moins car il est freiné par les forces de frottement. Les contraintes maximales se déplacent vers les zones de contact en raison des la présence, à cet endroit, des forces de frottement. Les configurations déformées, à l'instant $\mathrm{t}=2 \mathrm{~ms}$, sont représentées sur la figure 3 . La flèche située au centre des cylindres indique le sens d'évolution de ce dernier. On remarque que dans le cas A, le cylindre a rebondi alors que pour les cas avec frottement, le cylindre adhère sur les parois et cela d'autant plus que le coefficient de frottement est important. Toutefois, pour le cas $\mathrm{B}$, le cylindre arrive à décoller des parois et rebondit alors que dans le cas $\mathrm{C}$, le cylindre oscille en restant collé au parois. L'évolution du déplacement du centre du cylindre, noté $O$, est tracé (figure 4) en fonction du temps. Pour les trois cas, les figures 5, 6 et 7 montrent l'évolution de l'énergie cinétique $E_{c}$, de l'énergie de déformation élastique $E_{e}$ et de l'énergie totale $E_{t}$ en fonction du temps. Pour le cas A, le cylindre rebondit avec la même vitesse que la vitesse initiale, il n'y a pas de dissipation d'énergie comme le montre la figure 5. Pour le cas B, le cylindre rebondit mais avec une vitesse plus faible que la vitesse initiale, la figure 6 montre clairement la dissipation d'énergie. Lorsque le frottement devient encore plus important (cas C), le cylindre adhère aux surfaces de contact et l'énergie cinétique tend vers zéro, le cylindre reste bloqué (figure 7).
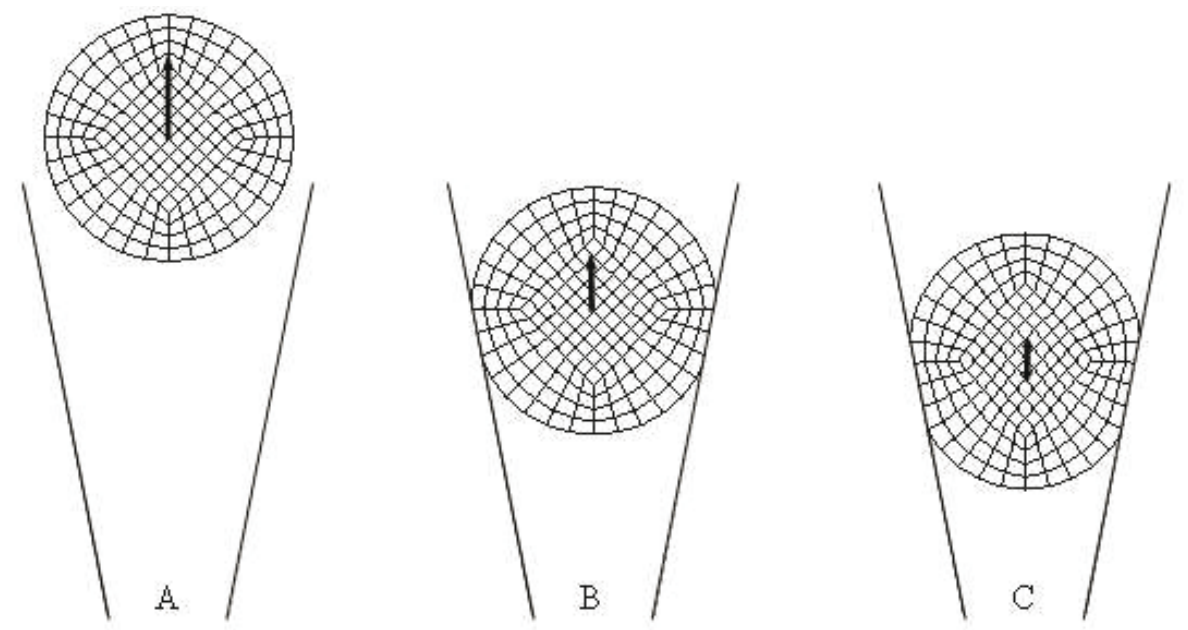

Figure 3. Configurations déformées à l'instant $t=2 \mathrm{~ms}$

On observe que l'énergie est parfaitement conservée dans le cas sans frottement (figure 5). Alors que dans les cas avec frottement, l'énergie totale décroît (figures 6 et 7). L'énergie totale est dissipée par frottement comme prévu. Il convient de souligner que l'énergie dissipée est calculée de manière quantitative. Il est également intéressant de savoir si l'énergie dissipée est proportionnelle au coefficient de frottement. Les résultats numériques indiquent que cela n'est pas le cas. Sur les figures 6 et 7, on constate que l'énergie dissipée est presque la même alors que les coefficients de frottement sont très différents. 

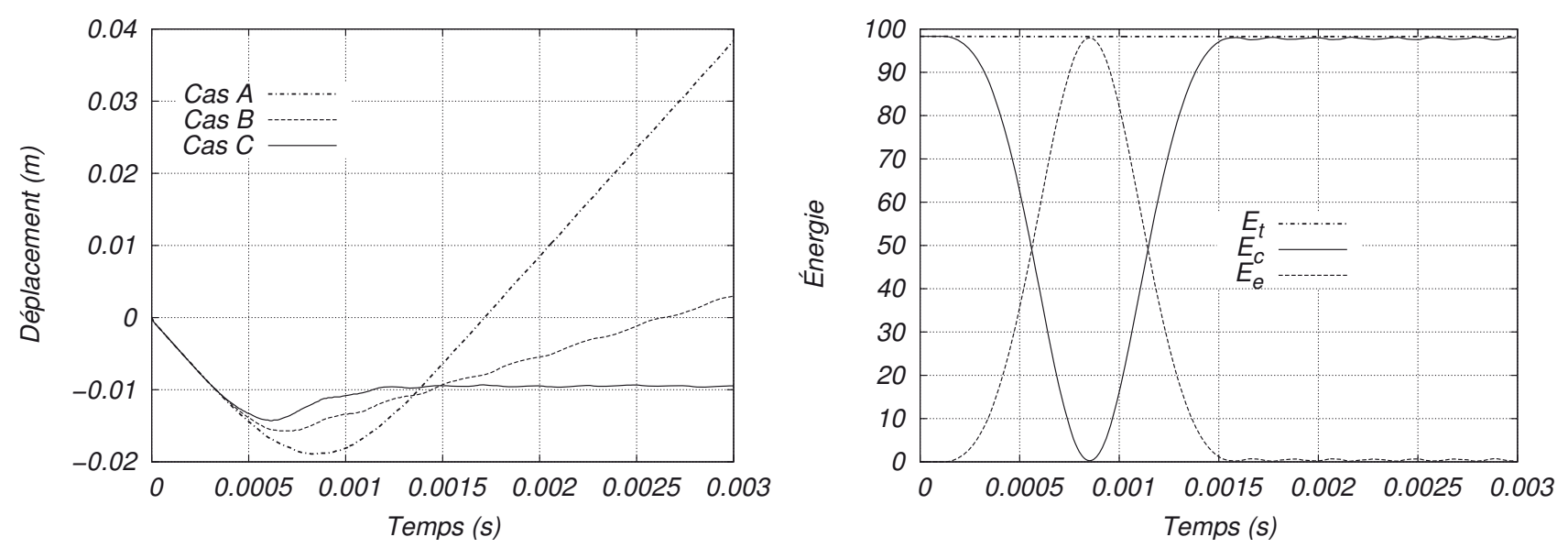

Figure 4. Déplacement du point $O$

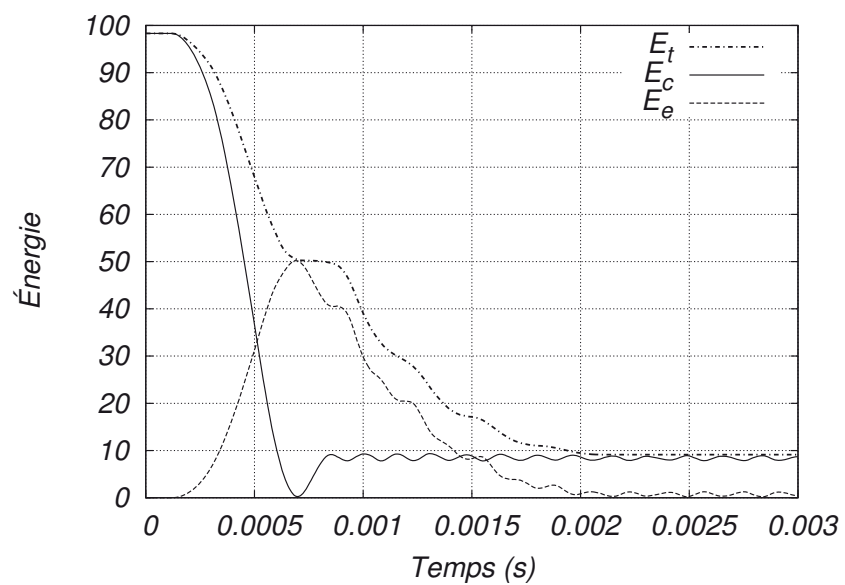

Figure 5. Évolution de l'énergie, $\mu=0,0$

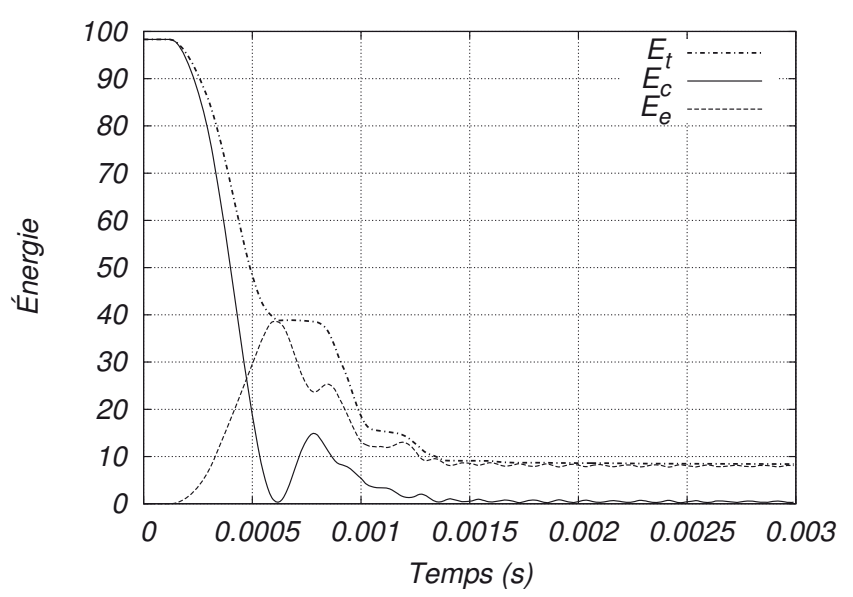

Figure 6. Évolution de l'énergie, $\mu=0,2$

Figure 7. Évolution de l'énergie, $\mu=0,4$

On constate un autre résultat intéressant sur cet exemple en comparant les cas B et C. Dans le cas B (figure 6), l'énergie cinétique tend vers une valeur constante et l'énergie de déformation élastique est égale à zéro après l'impact. Alors que dans le cas $\mathrm{C}$, (figure 7), l'énergie cinétique tend vers zéro et l'énergie de déformation élastique n'est pas libérée. Comme attendu, le cylindre est coincé entre les deux plans. Pour tous les cas, l'énergie totale reste presque la même. Pour cet exemple les simulations ont été réalisées sur un ordinateur équipé d'un processeur Pentium 4 fonctionnant à 2,8 Ghz. Le temps de calcul (en seconde) pour les différents cas sont : cas A; $62 \mathrm{~s}$, cas B; $77 \mathrm{~s}$ et cas $\mathrm{C} ; 83 \mathrm{~s}$. La prise en compte du frottement engendre une augmentation significative du temps de calcul.

\subsection{Quelques illustrations des autres possibilités du code}

Un échantillon de résultats démontre les capacités du code à résoudre divers types de problèmes. D'autres exemples sont disponibles en téléchargement sur la page web du laboratoire (Feng 2000). Les résultats sont visualisés à l'aide du logiciel de post- 
traitement FER/View (Feng et al. 2004) développé également au sein du laboratoire. Dans la suite, toutes les figures représentent la contrainte équivalente de von Mises.

Le premier exemple est l'impact d'un cylindre sur un bloc de caoutchouc qui repose sur un sol rigide (maillé avec un seul élément). Les vues prises à différents instants (figures 8 à 10) montrent que sous l'impact du cylindre le bloc se déforme et décolle du sol, le cylindre ayant quant à lui, rebondi.
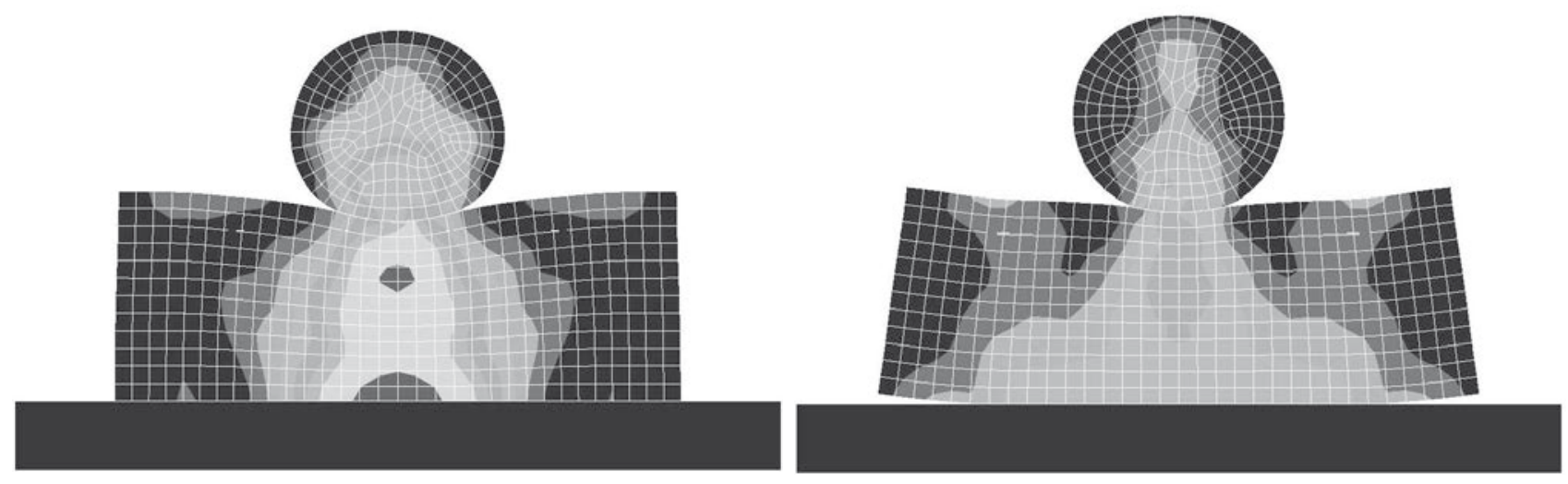

Figure 8. Le cylindre écrase le bloc

Figure 9. Le bloc se déforme et ses extrémités décollent de la paroi
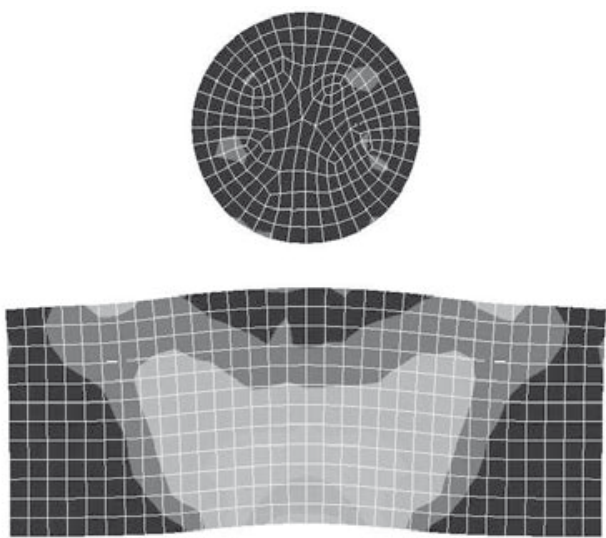

Figure 10. Le cylindre rebondit après l'impact, le bloc décolle complètement

Un autre exemple (figure 11) montre des contacts multiples entre des solides déformables dont le comportement est hyper-élastique (Feng et al. 2005b). Des cylindres sont enfermés dans une boîte dont une des parois est mobile. Un effort est exercé sur la paroi mobile qui vient déformer progressivement les différents cylindres. On observe le résultat sur la figure 12 qui montre également les contraintes équivalentes de von Mises sous forme d'iso-lignes. 

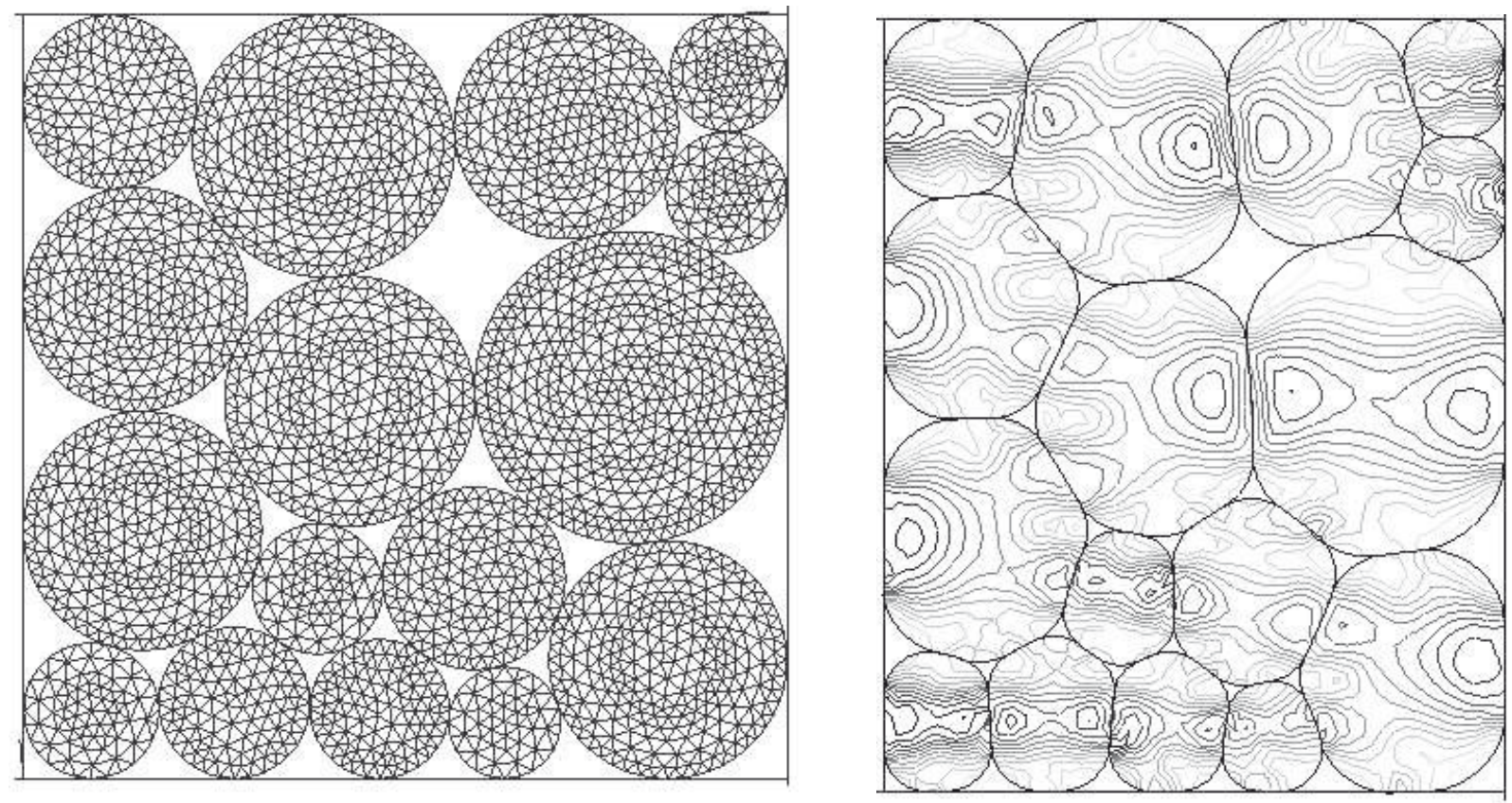

Figure 11. Multi-contact : maillage

Figure 12. Multi-contact : déformée

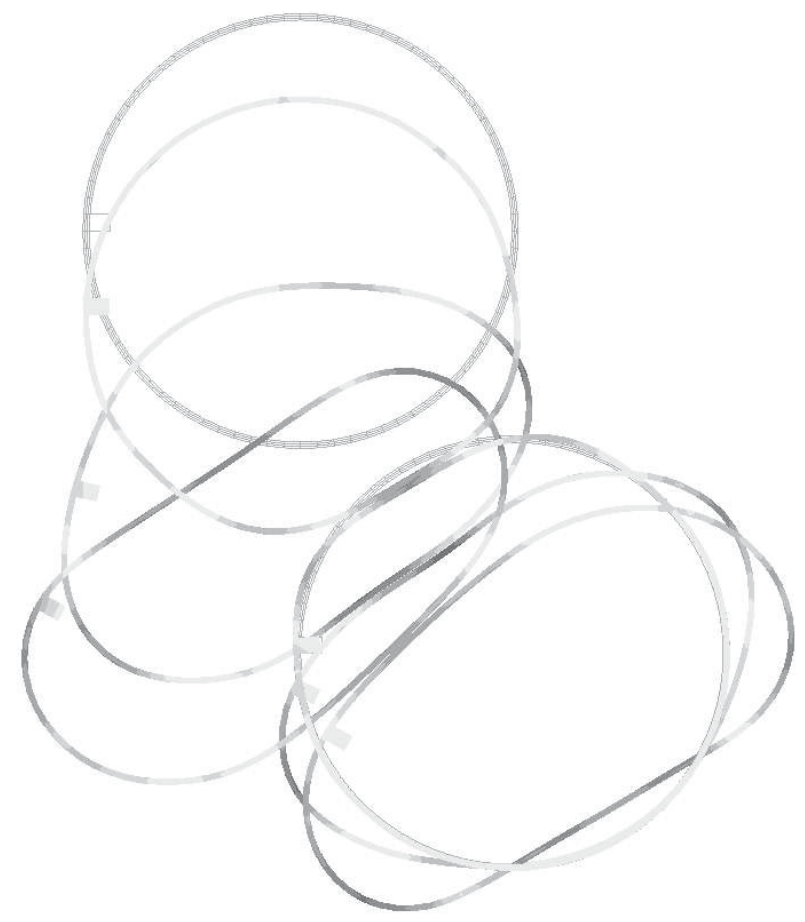

Figure 13. Impact entre deux anneaux élastiques

Un nouvel exemple reproduit une simulation déjà publiée (Laursen 2002) mais traitée par un algorithme de type Newmark avec réactualisation des vitesses. Un anneau élastique est lancé vers un autre anneau avec une vitesse initiale (figure 13).

Une petite excroissance a été ajoutée à l'intérieur des anneaux afin de bien visualiser la rotation de ces derniers. La figure 13 représente plusieurs instants de la simulation superposés, on peut visualiser également la contrainte équivalente de von Mises à l'intérieur de chaque anneau. Plusieurs phénomènes sont observés, impact 
des anneaux, roulement et glissement d'un anneau sur l'autre. En particulier, ce cas met en évidence les capacités du code à traiter les grands déplacements et les grandes rotations. De plus, on peut remarquer qu'il n'y a jamais pénétration d'un solide dans l'autre.

Enfin, le dernier exemple, tiré de (Love et al. 2003), consiste en l'impact de deux blocs élastiques 3D. Initialement les deux blocs sont distants mais ils ont leurs faces parallèles, le petit bloc est alors animé par une vitesse initiale ayant une composante normale et tangentielle. Suite au contact entre les deux solides, il s'ensuit une phase de glissement, mais le frottement entraîne le basculement du solide impacteur (figure 14).

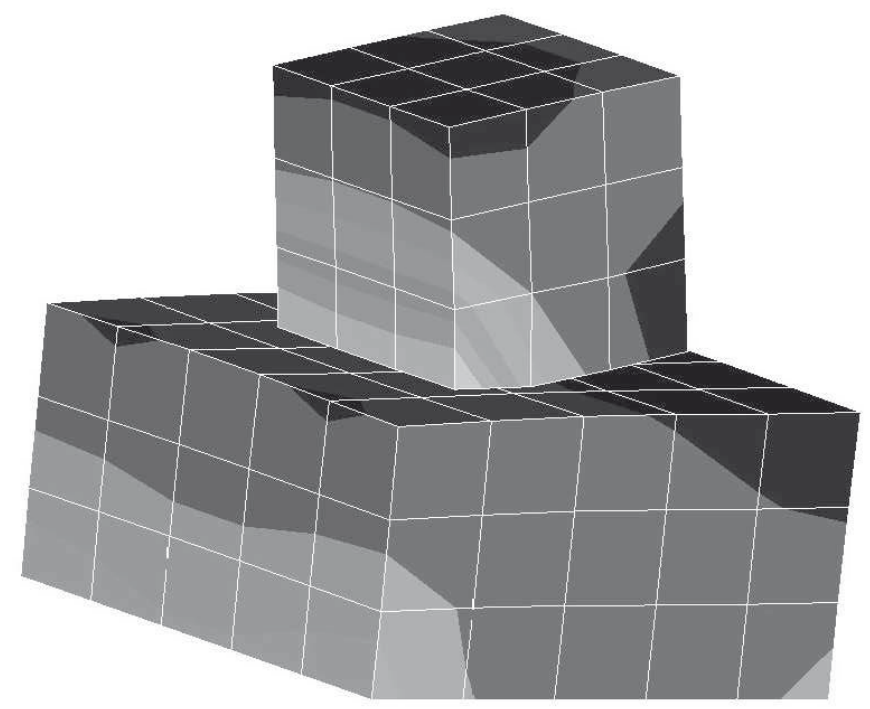

Figure 14. Impact de deux solides élastiques $3 D$

\section{Conclusion}

Cet article donne un aperçu des possibilités du code FER/Impact pour la simulation de problèmes de contact/impact avec frottement. Quelques exemples illustrent les performances de l'algorithme, notamment dans le cas de multi-contact entre solides déformables (élastiques ou hyper-élastiques).

Remerciements

Une partie des calculs a été réalisée sur les machines du CINES et de l'IDRIS.

\section{Bibliographie}

Armero F., Petocz E., « Formulation and analysis of conserving algorithms for frictionless dynamic contact/impact problems », Comp. Meth. Appl. Mech. Engng., vol. 158,, p. 269300, 1998. 
De Saxcé G., Feng Z.-Q., « New inequality and functional for contact with friction : The implicit standard material approach », Mech. Struct. \& Mach., vol. 19, p. 301-325, 1991.

De Saxcé G., Feng Z.-Q., " The bi-potential method : a constructive approach to design the complete contact law with friction and improved numerical algorithms », Mathematical and Computer Modeling, vol. 28(4-8), p. 225-245, 1998. special issue Recent Advances in Contact Mechanics.

Feng Z.-Q., « 2D or 3D frictional contact algorithms and applications in a large deformation context », Comm. Numer. Meth. Engng., vol. 11, p. 409-416, 1995.

Feng Z.-Q., « Some test examples of 2D and 3D contact problems involving Coulomb friction and large slip », Mathematical and Computer Modeling, vol. 28(4-8), p. 469-477, 1998. special issue Recent Advances in Contact Mechanics.

Feng Z.-Q., http ://gmfe16.cemif.univ-evry.fr :8080/ feng/FerSystem.html, 2000.

Feng Z.-Q., Cros J.-M., Magnain B., « Un algorithme efficace pour les problèmes d'impact avec frottement », Revue Européenne des Eléments Finis, vol. 14, n 1, p. 65-86, 2005a.

Feng Z.-Q., Feng Z., " FER/View - An interactive finite element post-processor », in Z.-H. Yao, M.-W. Yuan, W.-X. Zhong (eds), Computational Mechanics, WCCM VI in conjunction with APCOM04, Tsinghua University Press, Springer, 2004.

Feng Z.-Q., He Q.-C., Magnain B., Cros J.-M., « On the modelling of contact/impact problems between rubber materials », Contact Mechanics International Symposium (CMIS), Hannovre, 2005b.

Feng Z.-Q., Joli P., Cros J.-M., Magnain B., « The bi-potential method applied to the modeling of dynamic problems with friction », Comput. Mech., vol. 36, p. 375-383, 2005c.

Feng Z.-Q., Peyraut F., Labed N., « Solution of large deformation contact problems with friction between Blatz-Ko hyperelastic bodies », Int. J. Engn. Science, vol. 41, p. 2213-2225, 2003.

Jean M., « Dynamics with partially elastic shocks and dry friction : double scale method and numerical approach », 4th Meeting on unilateral problems in structural analysis, 1989. Capri.

Jean M., « The non-smooth contact dynamics method», Comp. Meth. Appl. Mech. Engng., vol. 177, p. 235-257, 1999.

Laursen T., Computational Contact and Impact Mechanics : Fundamentals of Modeling Interfacial Phenomena in Nonlinear Finite Element Analysis, Springer-Verlag, 2002.

Love G., Laursen T., « Improved implicit integrators for transient impact problems-dynamic frictional dissipation within an admissible conserving framework », Comp. Meth. Appl. Mech. Engng., vol. 192, p. 2223-2248, 2003.

Simo J., Wong K., « Unconditionally stable algorithms for rigid body dynamics that exactly preserve energy and momentum », Int. J. Numer. Meth. Engng., vol. 31, p. 19-52, 1991. 